\title{
A Modified Staged Continuous Tabu Search Algorithm*
}

\author{
Fengchen Wang ${ }^{1}$, Ronghua $\mathrm{Ji}^{2}$, Lijun $\mathrm{Qi}^{1}$ and Wanlin $\mathrm{Gao}^{2}$ \\ ${ }^{1}$ College of Engineering, China Agricultural University, Beijing, China \\ ${ }^{2}$ College of Information and Electrical Engineering, China Agricultural University, Beijing, China \\ wangfengchen@cau.edu.cn, jessic1212@cau.edu.cn
}

\begin{abstract}
Based on the staged continuous tabu search (SCTS) algorithm, a modified staged continuous tabu search (MSCTS) algorithm is proposed in this paper to improve the convergence, speed and robustness of tabu search (TS) algorithm. The improvements focus on the selection method of the neighborhoods in MSCTS algorithm. The generation of neighborhoods is guided by the multidimensional normal distribution function. In multidimensional normal distribution function, the mean value is the current optimal solution and the standard deviation is produced by the difference vector of the objective function at the current optimal solution. The range setting of neighborhood is different at different stage. 9 typical functions are used to test the performance of MSCTS and SCTS algorithm respectively. There are 5 indexes to evaluate the performance of both algorithms. The tests results show that MSCTS algorithm is good at dealing with the multivariate optimization problems. The calculated optimum solution for multi-variable function by MSCTS algorithm is about 5 to 17 times as near to the theoretical optimum solution as that of SCTS algorithm. As to the same test function, the calculation speed of MSCTS algorithm is about 3 to 17 times as many as that of SCTS algorithm. At the same time, the application of MSCTS algorithm is more extensive.

Index Terms - Global optimization, Tabu search algorithm, Neighborhoods structure, Selection range
\end{abstract}

\section{I . Introduction}

Optimization technology is widely applied in a variety of fields, such as engineering design, science analysis and financial data treatment. With computer technology getting increasingly powerful, optimization technology attracts more and more researchers' interests.

Tabu search (TS) algorithm is one of crucial global optimization techniques. TS algorithm was first proposed by Professor Glover in 1986, from University of Colorado [1-2], for solving combinatorial optimization problems. The essential characteristic of TS algorithm is that several optimal solutions found temporarily, called taboo objects, are recorded by tabu list, which prevents these taboo objects can be found again in next several iterations. The existence of tabu list persecutes computer to turn to another direction to find better solutions, so as to jump out of local optimal effectively.

Like other global optimization techniques, TS algorithm is applied in a variety of fields, too. In chemical engineering, TS algorithm was used to find good model parameters for metabolic flux analysis (MFA) problems [3]. This method was also reported to be used in facility layout to improve the efficiency of material handing within a manufacturing system
[4]. In addition, TS algorithm was reported to be used for structural software testing [5].

Although TS algorithm can get global optimum by tabu list, the probability of being trapped in a local optimum still exists. Many method were proposed to solve this kind shortage, such as using a specific neighborhood definition which employs a block of jobs notion [6], employing a flexible memory system to avoid the entrapment in a local minimum and developing the ideal of "distance" to the fitness to accelerate optimization [7], processing a new parallel model for TS algorithm based on the crossover operator of genetic algorithms [8], and using three continuous stages in process like stage continuous tabu search (SCTS) algorithm [9]. Each stage in SCTS algorithm will produce a starting point for next stage.

In this paper, we present a modified staged Continuous tabu search (MSCTS) algorithm based on SCTS algorithm. MSCTS algorithm employed new neighborhoods selection strategy using multidimensional normal distribution rule, new amnesty standards, and so on. We intend to prevent algorithm being trapped in a local optimum and achieve higher efficiency through those new rules.

\section{II . Overview of TS and SCTS Algorithm}

TS algorithm is good at resolving the global optimization problems. A general description of optimization problems is following:

$$
\begin{gathered}
\min F(s) \\
s \in N^{k} \text { and } N^{k}=\left\{a_{i} \leq x_{i} \leq b_{i}\right\}, i=1,2, \ldots, k
\end{gathered}
$$

Where $F(s)$ is the target function to be minimized, and $s=\left[x_{1}, x_{2}, \ldots, x_{k}\right]^{T}$, is the solution set. $a_{i}$ and $b_{i}$ are the boundary values.

The global optimum solution of the target function $F(s)$ will be calculated by TS algorithm.

In order to improve the algorithm efficiency, SCTS algorithm was proposed. There are three continuous stages and each stage is the standard TS algorithm. Each stage of SCTS algorithm produces a starting point $s_{0}$ for next stage to achieve the global optimum efficiently. The main differences among these three stages are the neighborhoods generation rules and the tabu list setting rules. Moreover, the tabu list in

\footnotetext{
* This work is partially supported by Chinese National Natural Science Foundation (31101609), and National Key Technology R\&D Program of China during the $12^{\text {th }}$ Five-Year Plan Period (Grand \#:2012BAJ18B07).
} 
each stage is independent of each other and is always reset at beginning of each stage.

\section{III . MSCTS Algorithm}

The general idea of MSCTS algorithm is similar to SCTS algorithm. MSCTS algorithm also consists of three stages and each stage is based on standard TS algorithm. We try to improve the performance of SCTS algorithm through changing the selection method of neighborhoods, and selection structure and selection range are taken into consideration.

\section{A. The Selection Structure of Neighborhood}

The multi-dimensional normal distribution function is proposed to generate neighborhoods in MSCTS algorithm at first and second stage. The center position of normal curve is decided by the mean value. The steep degree of normal curve is decided by the standard deviation. The steep degree of normal curve is decided by the standard deviation. In order to ensure that the current optimal solution was the center of neighborhoods, the current optimal solution is set as the mean value of multi-dimensional normal distribution function.

As for $\mathrm{N}$ dimension optimal problem, the current optimal solution $\mathrm{S}_{0}$ is $\mathrm{N}$ dimension vector. The gradient of the current optimal solution can reflect the changing trend of solution. At the same time, the dispersion degree of neighborhoods is reflected by the standard deviation of the normal distribution. The optimization results are archived faster if the current optimal solution is revised differently according to the change at different direction during optimizing, especially in first stage in MSCTS algorithm. So the standard deviation of multidimensional normal distribution function is set as the difference vector of $\mathrm{S}_{0}$ which is composed of the every dimension differences of objective function at $\mathrm{S}_{0}$. The changing trend of the objective function in each dimension will be reflected through the standard deviation vector. The larger the difference of one dimension is, the larger standard deviation of multidimensional normal distribution function at this direction is and the greater the possibility of the selected neighborhood objects at this direction far away the current optimal solution is. Actually, the difference vector of $S_{0}$ cannot be set as the standard deviation directly. The difference vector of $\mathrm{S}_{0}$ need be transformed.

All in all, at the first and second stage, the neighborhoods of $\mathrm{S}_{0}$ will be generated according to the multi-dimensional normal distribution function, whose the mean value is the current optimal solution and whose standard deviation is the transformed difference vector of $\mathrm{S}_{0}$.

At third stage, to improve the accuracy of the results, we use fixed step size to select neighborhoods during the one iteration. Every time when a current optimum solution which has not been tabooed is found, the step size will be narrowed at next iteration.

\section{B. The Selection Range at Different Stage}

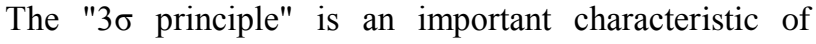
normal distribution. According to the " $3 \sigma$ principle", we can use the normal distribution function to select the neighborhoods which are able to cover almost the whole selection range. To accelerate algorithm and achieve the global optimum more efficiently, the selection ranges of neighborhood in three stages are set differently in MSCTS algorithm.

In the first stage, the neighborhoods range is the whole solution space so that the optimizing process can go through all the solutions. The approximate scope of optimum solution is fixed after the optimizing at the first stage.

Based on the optimization results of the first stage, the optimization in the second stage can be accelerated by narrowing the selection range of neighborhoods according to the current optimal solution. In the second stage, every time when a current optimum solution which has not been tabooed is found, the selection range will be narrowed. Besides, the optimal solution and its neighborhood selection range will be recorded. The latest optimal solution and its neighborhood selection range will be used in the third stage.

In the third stage, the selection range of neighborhood is fixed.

\section{IV . Parameter Optimization}

A. The shrinking ratio of search scope at the second stage (por)

The shrinking ratio of search scope at the second stage (por) is one of important parameters in MSCTS algorithm. To get reasonable por value, we did tests by using por $=[0.1,0.3$, $0.5,0.7,0.9]$ to determine that. Fig. 1 shows tests results.
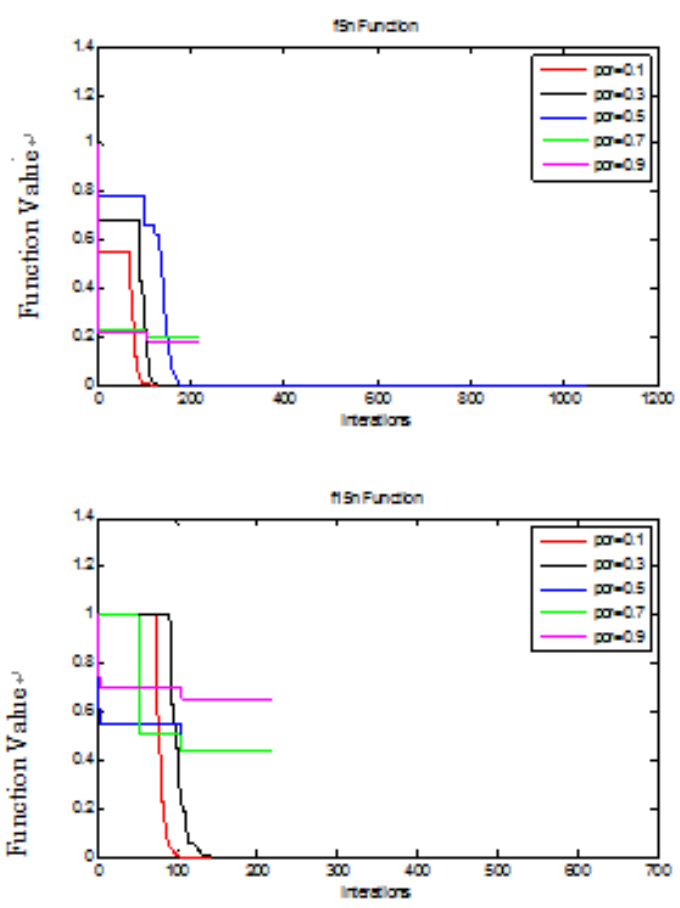

Fig. 1 The test results of por optimization

From tests results, we choose por $=0.1$ as the shrinking ratio of search scope at the second stage. 


\section{B. The shrinking ratio of step size at the third stage ( $p p)$}

The shrinking ratio of step size at the third stage $(p p)$ is also one of important parameters in MSCTS algorithm. To get reasonable $p p$ value, we did tests by using $p p=[0.1,0.3,0.5,0$. $7,0.9]$ to determine that, and we have run MSCTS algorithm to times for every $p p$ value. Fig. 2 shows tests results.
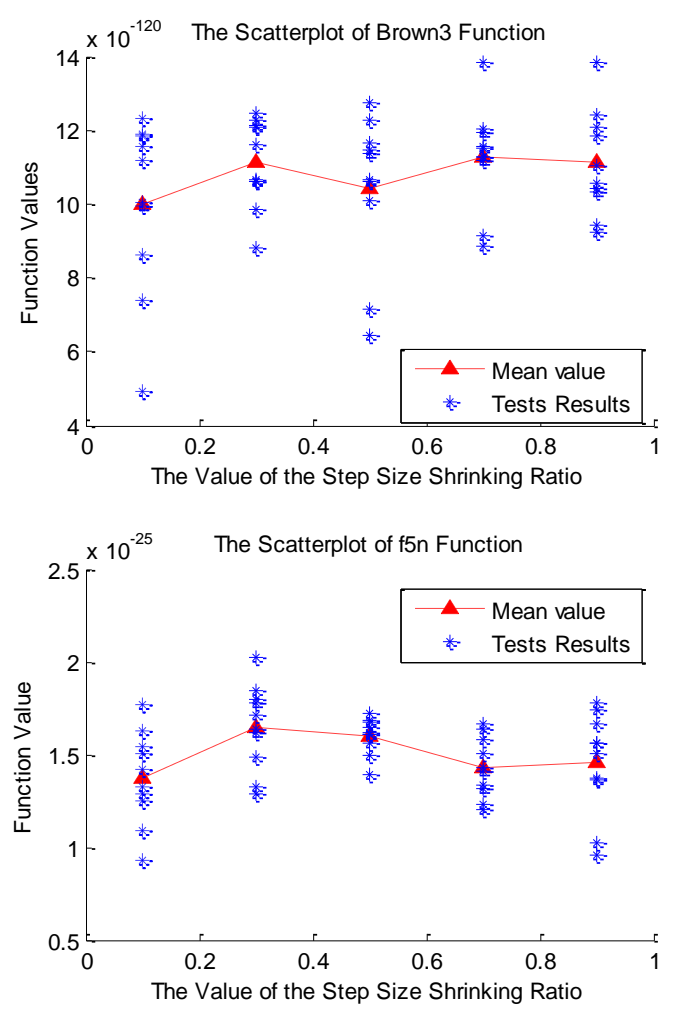

Fig. 2 The Scatter plot of test results for $p p$ optimization

From tests results, we choose $p p=0.1$ as the shrinking ratio of step size at the third stage.

\section{Computational Experiments and Comparative Analysis}

To demonstrate the effectiveness of MSCTS algorithm, we have not only done a great number of computational experiments, but also done the comparative analysis between MSCTS algorithm and SCTS algorithm in the convergence, speed, robustness and so on. The archived global optimum must be judged whether it is in the solution space because the neighborhoods are controlled by ' $3 \sigma$ principle' in the MSCTS algorithm.

\section{A. The Computational Experiment}

In order to test the performance of the MSCTS algorithm, a series of minima functions which were also used in the Ref. [9] were taken as test functions. The results of MSCTS algorithm were compared with those of SCTS algorithm. As for each test function, both algorithms have been run 10 times in once test at the same computer in order to get more reliable results.
The parameters setting of SCTS and MSCTS are listed in TABLE 1. The parameters of SCTS algorithm are set according to Ref. [9]. In the computational experiment, the performance of optimization algorithm is evaluated by 5 performance indexes which are described in TABLE 2. The experimental results are shown in TABLE 3.

TABLE 1 Parameter setting

\begin{tabular}{|c|l|c|}
\hline Parameter & \multicolumn{1}{|c|}{ Definitions } & Value \\
\hline Max_count & $\begin{array}{l}\text { The maximum iteration times at every stage } \\
\text { for our 9 test functions) }\end{array}$ & 4000 \\
\hline Max_kk & $\begin{array}{l}\text { The maximum iteration times of the current } \\
\text { optimum solution without any modification }\end{array}$ & 50 \\
\hline len & The length of tabu list & 5 \\
\hline$n u m$ & The number of neighborhoods & $5^{*} \mathrm{~N}$ \\
\hline$p p$ & $\begin{array}{l}\text { Shrinking ratio of search scope at the } \\
\text { second stage }\end{array}$ & 0.1 \\
\hline por & Shrinking ratio of step size at the third stage & 0.1 \\
\hline
\end{tabular}

TABLE 2 Performance Evaluating Indexes

\begin{tabular}{|c|l|c|}
\hline Index & \multicolumn{1}{|c|}{ Definitions } & \multicolumn{1}{|c|}{ Function } \\
\hline Max_value & $\begin{array}{l}\text { The maximum values selected from } \\
\text { results }\end{array}$ & $\begin{array}{c}\text { To evaluate the } \\
\text { ability of the } \\
\text { algorithm to } \\
\text { find the global } \\
\text { optimum } \\
\text { solution }\end{array}$ \\
\hline E_relative & $\begin{array}{l}\text { The minimum values selected from } \\
\text { results }\end{array}$ & $\begin{array}{l}\text { The relative error } \\
\text { The evaluate the } \\
\text { algorithm speed } \\
\text { once spent }\end{array}$ \\
\hline AIT & $\begin{array}{l}\text { The actual iteration times of the } \\
\text { algorithm }\end{array}$ & \multicolumn{1}{c}{} \\
\hline
\end{tabular}

TABLE 3 Experimental data of test function achieved by SCTS and MSCTS algorithm

\begin{tabular}{|c|c|c|c|c|c|c|c|c|}
\hline Function & $1^{\mathrm{a}}$ & 2 & & 3 & 4 & 5 & 6 & 7 \\
\hline \multirow{2}{*}{ Branin } & \multirow{2}{*}{2} & \multirow{2}{*}{0.379} & $\mathrm{M}^{\mathrm{b}}$ & 0.3979 & 0.3979 & 0 & 78 & 11 \\
\hline & & & $\mathrm{S}$ & 0.3979 & 0.3979 & 0 & 424 & 34 \\
\hline \multirow{2}{*}{$G P$} & \multirow{2}{*}{2} & \multirow{2}{*}{3} & $\mathrm{M}$ & 3 & 3 & 0 & 374 & 39 \\
\hline & & & $\mathrm{S}$ & 3 & 3 & 0 & 2112 & 181 \\
\hline \multirow{2}{*}{ Hart.34 } & \multirow{2}{*}{3} & \multirow{2}{*}{-3.863} & $\mathrm{M}$ & -3.863 & -3.863 & 0 & 837 & 154 \\
\hline & & & $\mathrm{S}$ & -1.001 & -3.863 & 0.02 & 4701 & 406 \\
\hline \multirow{2}{*}{ Shubert } & \multirow{2}{*}{2} & \multirow{2}{*}{$18 \overline{6} .73$} & M & -186.73 & -186.73 & 0 & 347 & 41 \\
\hline & & & $\mathrm{S}$ & -185.19 & -186.73 & 0.41 & 6060 & 339 \\
\hline \multirow{2}{*}{ Brown1 } & \multirow{2}{*}{20} & \multirow{2}{*}{2} & $\mathrm{M}$ & 2 & 2 & 0 & 1503 & 632 \\
\hline & & & $S$ & 2.0001 & 2 & $5 e-5$ & 1228 & 4415 \\
\hline \multirow{2}{*}{ Brown 3} & \multirow{2}{*}{20} & \multirow{2}{*}{0} & $\mathrm{M}$ & $5.27 \mathrm{e}-119$ & $9.04 \mathrm{e}-120$ & $3.09 \mathrm{e}-119$ & 2742 & 487 \\
\hline & & & $\mathrm{S}$ & $8.09 \mathrm{e}-5$ & $3.29 \mathrm{e}-5$ & $5.69 \mathrm{e}-5$ & 9593 & 2250 \\
\hline \multirow{2}{*}{$F 5 n$} & \multirow{2}{*}{20} & \multirow{2}{*}{0} & $\mathrm{M}$ & $9.18 \mathrm{e}-31$ & $1.93 \mathrm{e}-31$ & $5.57 \mathrm{e}-31$ & 992 & 458 \\
\hline & & & $\mathrm{S}$ & $1.31 \mathrm{e}-4$ & $2.32 \mathrm{e}-5$ & $7.71 \mathrm{e}-5$ & 9341 & 3500 \\
\hline \multirow{2}{*}{ F10n } & \multirow{2}{*}{20} & \multirow{2}{*}{0} & $\mathrm{M}$ & $1.68 \mathrm{e}-29$ & $5.46 \mathrm{e}-31$ & $8.67 \mathrm{e}-30$ & 833 & 419 \\
\hline & & & $\mathrm{S}$ & $9.8 \mathrm{e}-4$ & $3.63 \mathrm{e}-4$ & $6.72 \mathrm{e}-4$ & 9764 & 3536 \\
\hline \multirow{2}{*}{$F 15 n$} & \multirow{2}{*}{20} & 0 & $\mathrm{M}$ & $3.69 \mathrm{e}-15$ & $2.31 \mathrm{e}-24$ & $1.85 \mathrm{e}-15$ & 1472 & 383 \\
\hline & & & $\mathrm{S}$ & $6.96 \mathrm{e}-4$ & $3.61 \mathrm{e}-4$ & $5.28 \mathrm{e}-4$ & 9209 & 3463 \\
\hline
\end{tabular}

a 1 8: 1 The number of variables. 2 The theoretical solution of test functions. 3 Max_value. 4 Min_value. 5 E_relative. 6 AIT. 7 Time(s).

${ }^{\mathrm{b}} \mathrm{M}$ : MSCTS algorithm. S: SCTS algorithm.

B. The Analysis of the Results of Computation Experiment The results of the computation experiment are analyzed in 
terms of the convergence, the speed and the robustness.

\section{- Convergence}

MSCTS algorithm has better convergence for more complex problems. The convergence of MSCTS algorithm is evaluated by Max_value, Min_value and E_relative.

On the one hand, MSCTS algorithm can get more precise values. From TABLE 3, as for the test functions which have little variables, just like the Branin, Gold price, Shubert and Hartmann34, MSCTS and SCTS algorithm can both achieve the theoretical optimum solution in tests. However, when the number of variable of test function are more, like the Brownl, Brown3, F5n, F1On and F15n, the calculated optimum solution by MSCTS algorithm is much closer to the theoretical optimum solution than by SCTS algorithm.

On the other hand, MSCTS algorithm can be better in avoiding the local optimum. A test function is solved by an optimization algorithm, which repeats many times. The optimization algorithm is trapped by the local optimum if almost function values with the calculated optimization solution are same, and not the theoretical optimum value. However, if the calculated optimum values of the test function with little variables near the theoretical optimum value can be achieved, there is little possibility that algorithm is trapped by the local optimum.

\section{- Speed}

MSCTS algorithm can quickly solve all test functions. AIT and Time are used to evaluate the speed of these algorithms. From TABLE 3, for the same test function, the number of actual iteration times of MSCTS algorithm is 3 to 17 times as many as that of SCTS algorithm. Especially for Shubert which has many sharp peaks, the number of actual iteration times of MSCTS algorithm is 17 times as many as that of SCTS algorithm. The runtime of MSCTS algorithm is 3 to 9 times as many as that of SCTS algorithm. The larger the number of variables in test function can be resolved rapidly by MSCTS algorithm, which is a great improvement of MSCTS algorithm.

\section{- Robustness}

MSCTS algorithm can be applied in many fields than SCTS algorithm. The practicability of the MSCTS algorithm can also be described by E_relative. According to TABLE 3, SCTS algorithm cannot resolve the more complicated functions (like Brown3, F5n, F10n and F15n). However, little E_relative values were gotten by MSCTS algorithm. MSCTS algorithm can deal with practical problems in science and engineering efficiently.

\section{Conclusion}

In order to improve the convergence, speed and robustness of SCTS algorithm, MSCTS algorithm is proposed and modified at neighborhoods selection and setting range. The multidimensional normal distribution function is used to instruct the generation of the neighborhoods. The current optimal solution is taken as the mean value and the difference vector of the objective function as the standard deviation in the multidimensional normal distribution function.
The performance of MSCTS and SCTS algorithm are tested by 9 typical test functions respectively which results are contrasted and evaluated by 5 performance indexes. There are several conclusions based on the results and analysis.

It not only improves the convergence and robustness of MSCTS algorithm, but also the application range is expanded wildly because the neighborhood selection is guided by the change of the objective function at the current optimal solution. MSCTS algorithm works very well especially for complicated problem.

Due to different setting of neighborhood range at different stages, the calculating speed of MSCTS algorithm increase remarkably. The fastest optimizing speed of MSCTS algorithm can be improved to 17 times.

The problem of MSCTS algorithm is that the found optimum solution may go beyond solution space because the neighborhood selection is based on " $3 \sigma$ principle" of multidimensional normal distribution. In order to ensure the effectiveness of the optimum solution, the location of optimum solution should be judged by Point location in MSCTS algorithm.

\section{Acknowledgment}

We would like to thank R.T Zheng for his help to our development of this MSCTS algorithm. We also want to thank $\mathrm{Yu} \mathrm{Wu}$, who did much about testing our MSCTS algorithm, and all of people offered help to our study and writing this article. We would like to thank anonymous reviewers for their constructive comments and suggestions on this paper.

\section{References}

[1] Fred Glover, "Tabu Search-Part I", ORSA Journal on Computing, vol. 1, no. 3, Summer 1989.

[2] Fred Glover, "Tabu Search-Part II", ORSA Journal on Computing. vol. 2, no. 1, Winter 1990.

[3] Vahid Mansourkhaki, "Application of Tabu Search for Metabolic Flux Analysis Based on Labeling Balances", UMI Microform, 1439935, 2006.

[4] Wen-Hsing Liu, "Tabu Search Heuristics for the Dynamic Facility Layout Problem", UMI Microform, 1429106, 2005.

[5] Eugenia Díaza, Javier Tuya, Raquel Blancoa, José Javier Dolado, "A tabu search algorithm for structural software testing", Computers \& Operations Research, 35 (2008) 3052 - 3072, 2007.

[6] Eugeniusz Nowicki, Czestaw Smutnicki, "A fast tabu search algorithm for the permutation flow-shop problem", European Journal of Operational Research, 91 (1996) 160-175

[7] Whei-Min Lin, Fu-Sheng Cheng, and Ming-Tong Tsay, "An Improved Tabu Search for Economic Dispatch With Multiple Minima", IEEE Transaction on Power Systems, vol. 17, no. 1, February 2002

[8] Adem Kalinli, Dervis Karaboga, "Training recurrent neural networks by using parallel tabu search algorithm based on crossover operation", Engineering Applications of Artificial Intelligence, 17 (2004) 529-542

[9] R.T. ZHENG, N.Q. NGO, P. SHUM, S.C. TJIN, L.N. BINH, "A Staged Continuous Tabu Search Algorithm for the Global Optimization and its Applications to the Design of Fiber Bragg Gratings", Computational Optimization and Applications, 30, 319-335, 2005.

[10] Marcin Molga, Czesław Smutnicki, "Test functions for optimization needs", 3 kwietnia 2005

[11] R.T. ZHENG, N.Q. NGO, L.N. BINH, S.C. TJIN, "Two-stage hybrid optimization of fiber Bragg gratings for design of linear phase filters", Optical Society of America, vol. 21, no. 12/December 2004 